\title{
9 The Fourth Grade of Modal Involvement
}

\author{
Volker Halbach
}

\subsection{Four Grades of Modal Involvement}

Modalities are at the centre of many philosophical debates. They include apriority, analyticity, metaphysical, as well as logical and physical necessity, future and past truth, and being knowable or known. In philosophical logic the main tool for analyzing modal discourse is modal or intensional logic in the wide sense that includes temporal, epistemic, and deontic logic. The expressive weaknesses of modal logic have been known for a long time, and it is somewhat of an embarrassment of philosophical logic that the standard formalization of modality in its straightforward forms does not suffice as a framework for many well-known philosophical debates.

In this chapter I give some hints for developing formal languages with possible worlds semantics that can analyze modal discourse in its full force. Much of what I am going to say is tentative and experimental. This is because the project is huge: There is at least as much scope for work on this approach than on quantified modal logic. Therefore, I can only sketch some basic considerations. There are many aspects that will not be addressed at all. In particular, I will not say anything about extensions such as actuality operator, two-dimensional semantics, and so on.

I focus on metaphysical necessity as my main example of a modality; but the machinery sketched below is also adaptable to other modalities. Future and past truth should be straightforward, but epistemic modalities may be more difficult.

As a starting point, I revisit Quine's "Three grades of modal involvement" (1976, first published 1953). The three grades are three ways to view necessity, namely as a semantical predicate, a statement operator, or a sentence operator.

In modal logic modalities are conceived as operators: An operator $\square$ is combined with a formula $\varphi$ to obtain a new formula $\square \varphi$. The difference between statement and sentence operators is that the latter allow quantification into the scope of the modal operator. Thus a sentence operator 
$\square$ can be combined with a formula $\varphi$ containing free variables into a formula $\square \varphi$ that contains the same free variables as $\varphi$ itself. Quine's terminology is somewhat misleading, because sentences are understood here as open sentences with free variables. A statement operator does not permit quantification into its scope. De re modalities can be analyzed with sentence, but not with statement operators.

According to Quine, the lowest grade of modal involvement permits only 'semantic predicates': A semantic predicate Nec for necessity is combined with a singular term into a formula. ${ }^{1}$ Quine thinks of semantic predicates as applying to sentences, not propositions or other objects that Quine called 'creatures of darkness' elsewhere; but this is not the point here. It is rather that a semantic predicate can be applied to a variable: Nec $x$ is a formula with $x$ free.

Quine thought of semantic predicates as a light grade of modal involvement, but in another sense they are expressively richer than operators. Using modal predicates one can express quantifications. Quantified statements such as 'There are synthetic judgements a priori', 'All theorems of arithmetic are analytic', or 'There are necessary a posteriori truths' cannot be expressed using only an operator $\square$, at least not in a straightforward way. However, if modalities are conceived as predicates, quantified statements can be easily expressed: Kant's rejection 'There are synthetic judgements a priori' of empiricism, becomes the sentence $\exists x$ (Syn $(x) \wedge \operatorname{Apriori}(x))$ (omitting the restriction to judgements). ${ }^{2}$

For these reasons we require modal predicates. They are required to analyze the full force of modal discourse, especially in philosophy. With a semantic predicate for a modality comes the expressive power of what Quine called a 'statement operator'. That is, under fairly general assumptions everything that can be expressed using a statement operator $\square$ can be expressed also with a semantic predicate. As long as $\varphi$ does not contain $\square$, a sentence $\square \varphi$ can be replaced with $\operatorname{Nec}\ulcorner\varphi\urcorner$ where $\ulcorner\varphi\urcorner$ is a quotation or structurally descriptive name for the sentence $\varphi$. This induces a translation from the entire language with a statement operator to a language with a modal predicate only. However, this reduction or translation does not work for what Quine called 'sentence operators'. When a formula $\square \varphi$ with free variables is replaced with $\operatorname{Nec}\ulcorner\varphi\urcorner$, the free variables disappear; they are only mentioned, not used in $\operatorname{Nec}\ulcorner\varphi\urcorner$. In other words, Quine's semantic predicates do not permit de re modality.

Famously, Quine argued against de re modalities and sentence operators that apply to formulae with free variables-and failed completely at convincing the philosophical community to reject them. De re modalities are nowadays used without qualms almost everywhere; and it is hard to imagine many discussions in contemporary philosophy without the additional expressive strength gained from using de re modalities. Among the de re modalities analyzed in modal or intensional logics are temporal 
modalities (future and past truth), different epistemic modalities such as knowledge, and several varieties of necessity, especially metaphysical necessity. However, semantic predicates in Quine's sense do not suffice for analyzing de re modality; in particular, sentence operators are not reducible to semantic predicates.

I would not like to miss the expressive power of quantifying over the bearers of necessity or of quantifying into modal contexts. The full strength of modal discourse requires both: a predicate conception of modality and de re modality. None of Quine's three grades of modal involvement offers the strength of modal predicates and that of sentence operators. There is a conspicuous omission from Quine's list of grades of modal involvement: There are no predicates expressing de re modalities. ${ }^{3}$

One could hope that nevertheless semantic predicates together with sentential operators in Quine's sense would suffice: For quantified statements of the kind mentioned above a predicate, and for de re modality an operator that allows quantifying-in could be used. This is in itself unsatisfactory, because we need to switch between quantified modal logic for dealing with de re modalities and modal predicates for dealing with general quantified claims. But there is a class of statements for which both, an operator or de dicto predicate are insufficient. For this kind of statement the fourth grade of modal involvement is required. An example is the following claim:

(E) There is an object $o$ and there is a $P$ such that $P$ is necessary for $o$.

This claim may be taken as a statement of some kind of essentialism. Of course metaphysicians will disagree what kind of object $P$ can be: The traditional answer will be that $P$ must be a universal or property, while Quine and others might view $P$ as a formula.

The point is that neither a semantic predicate nor a sentential operator suffice to express (E) without further assumptions or additional devices. This can be seen as follows. If we use an operator as in modal logic, $\exists x \square P x$ fails as a formalization of (E), because it only claims that the specific property $P$ is necessary for some object. In order to quantify over $P$ we need a modal predicate (or higher-order quantification or the like). A unary predicate $\mathrm{Nec}$ for de dicto necessity may also be insufficient, but for a different reason: With a semantic predicate we cannot quantify any longer easily 'into the modal context', that is, over the objects that may have a property necessarily, at least if the semantic predicates apply to sentences. We may be able to express that some sentence of the form $P t$ is necessary, where $t$ is some closed term; but, of course, the claim (E) just says that $P$ is necessary for some $o$, whether $o$ has a name or not. ${ }^{4}$ Quine's semantic predicates cannot capture this higher kind of modal involvement. Discussions on various versions of essentialism, ante rem and in rebus conceptions of universals, and so on require 


\section{Volker Halbach}

de re modalities as predicates. Nothing should keep us from quantifying at the same time into modal contexts and over properties or predicates. Only the fourth grade of modal involvement affords this in a straightforward way.

In the following diagram the four grades of modal involvement are ordered by their strength. An arrow from one grade to another means that the grade at the origin of the arrow is stronger than that at the target, that is, the grade at the end of an arrow is reducible to that at its origin.

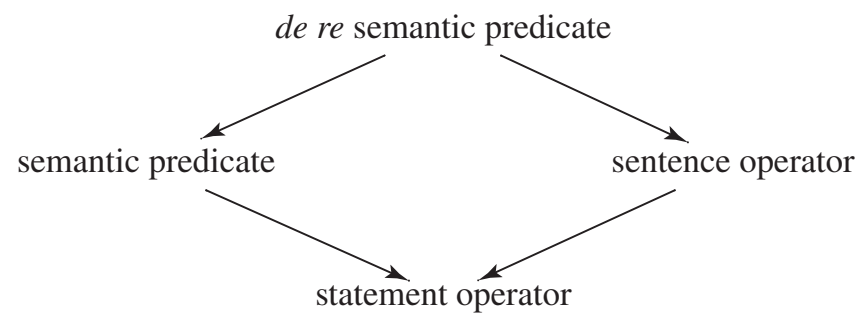

At the bottom, as the lowest form of modal involvement, is the conception of operator modal logic without any quantifying-in. ${ }^{5}$ As explained above, treating a modality as a semantic predicate or permitting quantifying-in increases the expressive strength. Neither is a semantic predicate for a modality reducible to the corresponding sentence operator nor vice versa: Semantic predicates and sentence operators add different kinds of expressive strength. The latter add de re modality, the former the possibility of quantifying over objects to which the modality is ascribed. At the top sits the highest, the fourth grade of modal involvement under which all others can be subsumed, a device that gives both kinds of expressive strength.

A formal framework for this fourth grade of modal involvement will need to deal not only with the traditional problems of modal predicates and de re modality as in quantified modal logic; there are also a few additional puzzles. There is not much literature on modal de re predicates. George Bealer's contributions $(1982,1993,1998)$ are probably the most sophisticated.

\subsection{De Re Semantic Predicates}

Different strategies have been tried to reach the fourth grade of modal involvement. One option is to use a sentence operator for necessity (permitting quantifying-in) and to expand the language with second-order order, substitutional, or propositional quantification. To emulate propositional quantification, quantifiers ranging over sets of possible worlds can be used, as suggested by Kripke (1959). Bull (1969), Fine (1970), 
Kaplan (1970), and subsequently many others elaborated on Kripke's suggestion. The simple approaches formulated over propositional logic have to be generalized to quantified modal logic to recover the strength of the fourth grade of modal involvement. This will yield some kind of second-order quantified modal logic as in Williamson (2013). I cannot provide a discussion of these approaches here. I refer the reader to Halbach and Leigh (2021) for a sketch of reasons why this approach is less promising than the strategy pursued here. One worry is that it will be difficult to express quantification over predicates of arbitrary arity: In higher-order logic one can quantify over propositions (0-place predicates), unary predicates, binary predicates, and so on; but simultaneously quantifying over all predicates of arbitrary arity is not easily possible without further resources.

Another option, with a similar problem, is the use of modal predicates of different arities: There would be a binary predicate that expresses that a single object has a necessary property or that a formula with one free variable is necessary for that object. A ternary predicate would be required for binary relations or formulae with two free variables, and so on. This becomes very clumsy, because there will be a necessity predicate for each arity (or one with variable arity). Moreover, we cannot easily express quantification over predicates with arbitrary arity. Examples of such quantifications will be given below.

The obvious solution consists in adapting Tarski's (1935) trick for truth to modalities. Tarski used a satisfaction predicate applying to formulae and variable assignments to define the unary truth predicate. Corresponding to the truth predicate we have the unary de dicto predicate for necessity; and corresponding to the binary satisfaction predicate, applying to formulae and variable assignments, we have the binary de re necessity predicate applying to formulae (or universals) and variable assignments.

A language with such a binary modal predicate is expressively richer than one with modal predicates for each arity. By quantifying over formulae (or corresponding universals) and variable assignments we are not restricted to a fixed arity. For instance, we can express essentialist claims in the style of (E) above in a more cautious way by saying that some objects necessarily stand in some relation. With this formulation we do not commit ourselves to a relation of a specific arity. By using a binary predicate applying to predicates of arbitrary arity and sequences of objects, this can be expressed with the formula $\exists x \exists y \operatorname{Nec}(x, y)$ or $\exists x \exists y(\operatorname{For}(x) \wedge \operatorname{As}(y) \wedge \operatorname{Nec}(x, y))$, where $\operatorname{For}(x)$ expresses that $x$ is a formula (relation) and As $(y)$ that $y$ is a variable assignment.

Using a predicate like Nec we can also express de re factivity. This is the claim that if a formula (or relation) is necessary of some objects, then it is true of those objects. This can be parsed as follows: If a formula $x$ is necessary of a string $y$ of objects, then $x$ is satisfied by $y$, or formally 


\section{Volker Halbach}

$\forall x \forall y(\operatorname{Nec}(x, y) \rightarrow \operatorname{Sat}(x, y))$. Again the quantifiers may be restricted to formulae and assignments as above. The factivity of de re knowledge can be expressed by replacing the binary predicate for necessity with a corresponding binary predicate for de re knowledge. De re factivity implies de dicto factivity, that is, the claim that whatever is necessary is true, but the converse implication does not hold.

At this point it is worth emphasizing the following: I am far from claiming that binary modal predicates of the kind described above are close to the methods of expressing the fourth degree of modal involvement in natural languages. A linguistic analysis is not the target of the present paper, although there are clearly some very interesting differences between modalities in the natural languages. For instance, temporal notions are usually expressed in the languages with which I am familiar by modifying the verb, either directly or with an auxiliary verb. The tense of a verb is probably most faithfully represented in a formal language by a tense operator, that is, a sentence operator in Quine's sense, not by a primitive predicate for future or past truth. Moreover, in many cases modalities can be expressed in many different ways in natural languages. We have modal operators such as necessarily as well as predicate phrases such as is necessary in English. I aim to provide a formal framework that allows us to analyze philosophical modal discourse in its full strength; this requires quantification over universals or formulae and variable assignments without fixed arity. If possible, I prefer to be parsimonious and not introduce devices that are reducible to binary predicate for de re modalities, even if there are analogues of these devices in natural languages.

All my claims so far about the strength of various devices for expressing modalities rely on appeals to some informal semantics. Of course, a plethora of various semantic systems exists for modal logics; but they are largely missing for corresponding binary predicates. Therefore, to substantiate my claims and to assess the prospects of analyzing de re modalities using binary predicates of the kind described, a formal semantics for languages with such predicates are needed.

\subsection{Possible Worlds Semantics}

In this section I extend and adapt the possible worlds semantics for a unary modal predicate developed by Asher and Kamp (1989), Halbach et al. (2003), Halbach and Welch (2009), and Halbach and Leigh (2021) to a semantics for the binary predicate $\operatorname{Nec}(x, y)$. I think of the semantics given below as a starting point. Several assumptions may be tweaked. Moreover, the account below can be generalized in many ways to different modalities and multimodal settings.

As pointed out at the end of the previous section, a formal semantics is needed in order to substantiate various claims about the strength of 
modal devices such as the binary modal predicate Nec, unary semantic predicates, the operator $\square$ of modal logic as sentence and statement operators, and perhaps further expressions. This requires a certain degree of adequacy of the semantics for which I will not argue.

There are further reasons for developing possible worlds semantics for Nec. In particular, I would like to transfer insights that have been obtained using possible worlds semantics for the modal operator $\square$. Giving up possible worlds semantics together with the operator conception of modality would be a huge loss. I do not intend to reject sentence and statement operators. My complaint is that they cannot capture the full force of modal talk; but as long as this force is not required, modal operators and their accompanying possible worlds semantics can shed light on many questions and puzzles.

The aim of this section is to define possible worlds models such that the formula $\operatorname{Nec}\left(\left\ulcorner\varphi\left(x_{1}, \ldots, x_{n}\right)\right\urcorner, a\right)$ holds at a world $w$ iff $\varphi\left(x_{1}, \ldots, x_{n}\right)$ holds at all worlds $v$ with $w R v$, if the free variables $x_{1}, \ldots, x_{n}$ are assigned values $a\left(x_{1}\right), \ldots, a\left(x_{n}\right)$. Here $\left\ulcorner\varphi\left(x_{1}, \ldots, x_{n}\right)\right\urcorner$ is a name for the formula $\varphi\left(x_{1}, \ldots, x_{n}\right)$, and $a\left(x_{k}\right)$ the value assigned to the variable $x_{k}$ by $a$. I will be sloppy with notation and, for instance, use $a$ in the object language, even though we might lack a name for it.

When setting up possible worlds semantics for $\mathrm{Nec}$, the same choices as for quantified operator modal logic have to be faced, and, as is wellknown, there are many (see, for instance, Garson, 2001). In particular, we have to decide whether to opt for a possibilist treatment of quantifiers with a fixed domain for all worlds or for actualism with domains that can vary between world.

There are also decisions that do not arise in quantified operator modal logic. On the predicate approach, we can quantify over the objects that can be necessary and they can inhabit possible worlds along with other objects (unlike propositions conceived as sets of possible worlds, which cannot themselves be elements of a world). Which of these objects exist would differ from world to world, if we decided to ascribe necessity to sentence tokens. I think of them as types and assume that they exist in all worlds. Metaphysical questions and decisions of this kind are suppressed in the usual variants of quantified modal logic; on the predicate approach they can be addressed in the object language.

Finite sequences of objects are needed as variable assignments for de re modalities. I assume that all worlds are closed under the formation of sequences. This is again a strong ontological assumption, which I endorse for metaphysical necessity, but not for other modalities. Only objects existing in the world can form part of a sequence in that world. I discuss potential problems arising from this assumption after having introduced formal possible worlds semantics for languages with modal predicates. 
For my purposes here there is no need to be very specific about the language. The language should contain vocabulary that allows one to talk about either expression in the language or corresponding 'universals'. I have a preference for expansions of languages described in Halbach and Leigh (2021), but expansions of arithmetic or set theory are equally suitable. The language should provide vocabulary for talking about syntax or universals, finite sequences of objects, and possibly other 'ordinary' objects. I use the syntactic approach and terminology. The reader preferring universals will have to replace 'sentence' with 'proposition', 'formula with one free variable' with 'property', and so on. Given that we need arbitrarily long finite sequences of arbitrary objects as variable assignments anyway, we can understand expressions as a specific kind of such sequences, namely those with symbols as members of the sequence.

Each expression in the non-logical vocabulary falls into at least one of three groups:

1. The syntactic vocabulary contains at least a unary predicate that applies exactly to all expressions of the language. Quantifiers relativized to this predicate range exactly over all syntactic objects. The other syntactic vocabulary may express operations such as concatenation and substitution and allow one to define grammatical categories such as variables, predicate expressions, connectives, etc.

2. The sequence vocabulary includes again a relativizing predicate that applies exactly to finite sequences. Further vocabulary may express concatenation of finite sequences, projections, etc.

3. The 'contingent' vocabulary contains all remaining non-logical expressions. A relativizing predicate is not required, because 'contingent' objects are exactly those that are not syntactic or sequences.

As mentioned above, it is not assumed that finite sequences and expressions do not overlap. However, the contingent objects are exactly those that are neither expressions nor sequences. The use of the term 'contingent' may be somewhat misleading. It only means that no assumptions about the interpretation of the contingent vocabulary are made in possible worlds semantics. This does not rule out that the contingent vocabulary is interpreted at all worlds in the same way in a given model. For instance, some vocabulary of pure mathematics may form part of the contingent vocabulary, and we may confine our attention to interpretations that assign the same extensions to these expressions at all possible worlds. But this restriction is not imposed by possible worlds semantics, but rather by other considerations.

The following definition of a pre-model is close to the definition of a possible worlds model in modal logic, the main difference being that it is assumed that all expressions exist in every world and that worlds are closed under the formation of finite sequences. 
DefinItIon 9.3.1. A triple $\langle W, R, I\rangle$ is a pre-model iff

1. $W$ is a non-empty set.

2. $R$ is a binary relation on $W$.

3. $I$ is a function that assigns each world $w \in W$ a domain $\mathcal{D}_{w} . \mathcal{D}_{w}$ contains (codes of) all strings of $\mathcal{L}_{N}$-symbols, possibly further 'contingent' objects and all finite sequences of all objects in $\mathcal{D}_{w}$. Moreover I provides interpretations of the contingent vocabulary over the domain $\mathcal{D}_{w}$.

Of course $\langle W, R\rangle$ is a frame in the usual sense in modal logic. Each world contains contains all $\mathcal{L}_{N}$-expressions and therefore infinitely many objects. Moreover, finite sequences can be formed from all objects in the world. Sequences themselves can be elements of sequences. Therefore each domain $\mathcal{D}_{w}$ is closed recursively under the formation of sequences. I do not make any assumption on whether expressions conceived as sequences of symbols are identical with these finite sequences.

Consequently, the domain $\mathcal{D}_{w}$ of each world $w \in W$ consists of three types of objects:

1. $\mathcal{D}_{w}$ contains all strings of symbols.

2. $\mathcal{D}_{w}$ contains arbitrarily many further 'contingent' objects.

3. $\mathcal{D}_{w}$ contains all finite sequences of objects from 1,2 , and 3 . This includes mixed sequences that have syntactic, contingent, and finite sequences as entries. Thus the domain of each world is defined recursively.

A pre-model provides interpretations for the entire language at each world $w \in W$, except for the binary necessity predicate Nec. At any world the syntactic and sequence vocabulary is interpreted in the standard way. This means that the syntactic vocabulary is interpreted in the same way at all worlds. However, worlds differ with respect to the objects that exist in them and consequently also with respect to the sequences that exist in them. Thus the interpretation of the vocabulary about sequences at a world depends only on the domain $\mathcal{D}_{w}$ of that world, because only sequences whose ultimate components exist at that world also exist at that world.

If $\varphi$ is a formula not containing the modal predicate $\mathrm{Nec}$ and $a$ a variable assignment over $\mathcal{D}_{w}$, I write $\langle W, R, I\rangle \vDash_{w} \varphi[a]$ to indicate that the formula $\varphi$ is satisfied by the variable assignment $a$ at world $w$. As mentioned above, variable assignments are conceived as finite sequences and thus the question arises about situations where $\varphi$ contains variables outside the domain of $a$. I could rule this out without real loss of generality, but prefer to stipulate that $a(x)$ is always a certain expression, say $\neg$ 
and thus effectively make every variable assignment a total function from the set of variables. By our stipulations, $\neg$ is in the domain of every world.

What is missing from this account is an interpretation of the de re modal predicate Nec. The interpretation $B$ of the binary predicate symbol Nec is provided separately from $I: B$ is a function that assigns to each world a binary relation (set of ordered pairs) on the set of objects that exist at $w$. I write $\langle W, R, I, B\rangle \vDash_{w} \varphi[a]$ iff $\varphi$ holds at world $w$ under the variable assignment $a$ if $\mathrm{Nec}$ is interpreted according to $B(w)$. I now define what a possible worlds model ('pw-model' for short) is.

Definition 9.3.2. $\langle W, R, I, B\rangle$ is a pw-model iff $\langle W, R, I\rangle$ is a pre-model and $B$ a function satisfying the following properties:

(i) $B$ is a function assigning to each world $w \in W$ a set of pairs $\langle\varphi, a\rangle$ such that $\varphi$ is a formula and $a$ is a finite sequence in $\mathcal{D}_{w}$.

(ii) $\langle\varphi, a\rangle \in B(w)$ iff for all $v$ (if $w R v$ then $\langle W, R, I, B\rangle \vDash_{v} \varphi[a]$ ).

In (ii) the variable assignment $a$ in [a] belongs to the metatheory, while pair $\langle\varphi, a\rangle$ is a possible element of the extension $B(w)$ of Nec at $w$ and thus $a$ an element of $\mathcal{D}_{w}$. That is, variable assignments belong to both, the object and metatheory.

Condition (ii) of Definition 9.3.2 forces the interpretation of $\mathrm{Nec}$ as truth in all accessible worlds. Suppressing the variable assignment, it means that a sentence $\varphi$ is in the extension of $\mathrm{Nec}$ at a world $w$ iff $\varphi$ is true in all worlds accessible from $w$. By condition (i), if $\langle\varphi, a\rangle \in B(w)$, then $a \in \mathcal{D}_{w}$ and therefore $a(x) \in \mathcal{D}_{w}$ for all variables $x$. That is, a variable assignment in a world $w$ assigns variables only values that exist in $w$. But of course $a(x)$ need not exist in another world, that is, we may have $a(x) \notin \mathcal{D}_{v}$ for some other world $v \neq w$. In particular, when we pass from a world $w$ to all worlds $v$ that can be seen by $w$, a variable assignment with $\langle\varphi, a\rangle \in B(w)$ might assign a value to the variable $x$ that is not in $\mathcal{D}_{v}$. Thus we need to make a stipulation about how to understand the right-hand-side of (ii), that is, $\langle W, R, I, B\rangle \models_{\nu} \varphi[a]$ if $\varphi$ contains a free variable $x$ such that $a(x) \notin \mathcal{D}_{v}$. The situation is similar to free logic and individual constants that do not denote. There are various options. Here I adopt the policy of negative free logic. If $\varphi$ is an atomic formula with a free variable $x$ such that $a(x) \notin \mathcal{D}_{v}$, then $\langle W, R, I, B\rangle \not \#_{v} \varphi[a]$. For instance we have $\langle W, R, I, B\rangle \not \not_{v} x=x[a]$. Of course, we still have $\langle W, R, I, B\rangle \models_{\nu} \forall x x=x[a]$ as quantifiers range over objects in $\mathcal{D}_{v}$.

The situation is different from ordinary quantified modal logic, because we have variable assignments not only in the metatheory (as in quantified modal logic), but we are also talking about variable assignments in the object language. In $\langle W, R, I, B\rangle \models_{\nu} \varphi[a]$ the variable assignment $a$ is in the metatheory of course, while in $\exists y \operatorname{Nec}(x, y)$ we are quantifying over variable assignments in the object language. The 
metatheory is extensional. Every variable assignment that exists at some world is also available in the metatheory. But of course not every variable assignment in our metatheory exists in every world: only if all values exist in $w$, the variable assignment exists in $w$.

In the usual possible worlds semantics for modal operators, one defines recursively the semantics for $\square$ from the interpretation of the other vocabulary at each world. Definition 9.3.2, in contrast, does not imply that for every pre-model $\langle W, R, I\rangle$ there is exactly one $B$ such that $\langle W, R, I, B\rangle$ is a pw-model. In fact, both, existence uniqueness fail. Halbach et al. (2003) and Halbach and Leigh (2021) provide more information on existence and uniqueness conditions. I mention only some observations that apply to the present framework.

A relation $R$ is converse wellfounded iff there is no infinitely ascending sequence $w_{1} R w_{2} R w_{3} \ldots$ of objects.

LEMmA 9.3.3. If the accessibility relation $R$ of a pre-model $\langle W, R, I\rangle$ is converse wellfounded on $W$, then there is a unique $B$ such that $\langle W, R, I, B\rangle$ is a pw-model.

The lemma is proved by defining $B$ on all $w \in W$ inductively on the converse of $R$, starting from the dead ends of $R$, that is, worlds $w \in W$ such that there is no $v \in W$ with $w R v$. At a dead end $w \in W$, we have $\langle\varphi, a\rangle \in B(w)$ for all formulae $\varphi$ and variable assignments $a \in \mathcal{D}_{w}$, because trivially $\langle W, R, I, B\rangle \vDash_{v} \varphi[a]$ for all worlds $v$ with $w R v$, as there are no such worlds. Once $B(v)$ has been defined for all worlds $v$ with $w R v$, we can set

$$
B(w):=\bigcap_{w R v}\left\{\langle\varphi, a\rangle:\langle W, R, I, B\rangle \models_{v} \varphi[a]\right\} \cap \mathcal{D}_{w}
$$

The intersection with $\mathcal{D}_{w}$ ensures that we only include variable assignments that exist at world $w$. If $R$ is converse wellfounded, this definition fixes $B(w)$ for all $w \in W$.

Lemma 9.3.3 gives us a sufficient condition for the existence of a pwmodel $\langle W, R, I, B\rangle$ based on a given pre-model $\langle W, R, I\rangle$. I turn now to necessary conditions.

Under fairly general circumstances, there cannot be a pw-model $\langle W, R, I, B\rangle$ with a reflexive or symmetric accessibility relation $R$. This is a direct consequence of the paradoxes. Since $\varphi$ is a sentence, the variable assignment $a$ is irrelevant and $\forall a \operatorname{Nec}(\ulcorner\varphi\urcorner, a)$ expresses de dicto necessity of $\varphi$ in the same way the unary truth predicate can be defined as the satisfaction of a sentence by all variable assignments. If $R$ is reflexive, we have the following for all sentences $\varphi$ and worlds $w \in W$, as in operator modal logic:

$$
\langle W, R, I, B\rangle \models_{w} \forall a \operatorname{Nec}(\ulcorner\varphi\urcorner, a) \rightarrow \varphi
$$

Using the diagonal lemma, a sentence $\lambda$ can be obtained such that $\lambda \leftrightarrow \neg \forall a \operatorname{Nec}(\ulcorner\lambda\urcorner, a)$ holds at all worlds. Of course, this is the liar 
sentence for the 'truth predicate' $\forall a \operatorname{Nec}(x, a)$. Combining this with (9.1) for $\lambda$ yields the following for all $w \in W$ :

$\langle W, R, I, B\rangle \models_{w} \lambda$

Since $\lambda$ holds at all worlds, $\forall a \operatorname{Nec}(\ulcorner\lambda\urcorner, a)$ holds at all worlds. This clashes with following consequence of the diagonal property of $\lambda$ :

$$
\langle W, R, I, B\rangle \models_{w} \neg \forall a \operatorname{Nec}(\ulcorner\lambda\urcorner, a)
$$

This contradiction is only a variant of Montague's (1963) theorem, as explained in Halbach and Leigh (2021). This can be generalized as follows:

Theorem 9.3.4. If the relation $R$ is converse illfounded on $W$, the syntactic vocabulary sufficiently expressive, and the contingent vocabulary contains at least one sentential parameter, then there is a $I$ such that $\langle W, R, I\rangle$ is a pre-model, but there is no $B$ such that $\langle W, R, I, B\rangle$ is a pw-model.

For a detailed proof the reader is referred to Halbach and Leigh (2021). First it is shown that one can define the modal predicate Nec* associated with the transitive closure of $R$. Using the diagonal lemma one proves that Löb's theorem holds for this predicate Nec*. Löb's theorem is of course a principle of transfinite induction that may fail if $R^{*}$ is not converse wellfounded. To show this, one can choose an interpretation $I$ that makes the sentential parameter true at all converse wellfounded worlds, but false at the converse illfounded worlds. If there is no contingent vocabulary (the sentential parameter in the theorem), there can be a converse illfounded $R$ such that $\langle W, R, I, B\rangle$ is a pwmodel and the situation becomes complicated. See Halbach et al. (2003).

\subsection{The Challenge of the Paradoxes}

Theorem 9.3.4 means that the accessibility relation cannot be total on all worlds; and we cannot expect modal predicates to satisfy the schemata of the modal systems T, B, S4, or S5 for all sentences (especially those sentences without equivalent in ordinary modal operator logic such as sentences obtained by Gödel's diagonal lemma). If we are confined to converse wellfounded frames for possible worlds semantics of predicates, then one might suspect that we cannot have a reasonable semantics for $\mathrm{Nec}$ as metaphysical necessity. Perhaps the accessibility relation need not be total and, perhaps, not even transitive for metaphysical necessity; but at last it should be reflexive. This is ruled out by Theorem 9.3.4.

The reader may wonder whether this shows that the predicate approach is to be rejected. In the light of the paradoxes Montague (1963) rejected 'syntactic' treatments of modality, that is, treatments of modality as predicates of sentences. The restrictions in Theorem 
9.3.4 only spell out the consequences of the paradoxes as restrictions on the accessibility relations and they thereby systematize them. Should one agree with Montague, abandon predicate approaches, and resort to operator modal logic? The price for this move will be a significant reduction in expressive power of our language. This matters most to philosophers, who are interested in the quantified claims mentioned in the first section.

The situation can be compared with that for truth: We can reject the predicate conception of truth and opt for an operator treatment. This will result the trivial modal logic with $\square \varphi \leftrightarrow \varphi$ as characteristic axiom. Although this move immediately blocks the paradoxes, it has not been very popular. What saved truth from the fate of metaphysical necessity and other modalities is that the truth predicate becomes trivial and boring, once it is stripped of its ability to express generalizations. When metaphysical necessity is treated in the same way, there are still many interesting things to say. But that is not a good justification for accepting the weakening of expressive power caused by the transition from $\square$ to Nec.

Rejecting modal predicates because of the paradoxes is as sensible as rejecting the theory of distances because of Zeno's paradoxes and rejecting set theory because of Russell's and Burali-Forti's paradox. We may have to revise or refine some of our naive expectation, but we will gain expressive strength in return. This strength is needed for general claims in philosophy. If we give it up in favour of modal operators, the way philosophy is done will have to be changed profoundly. Therefore, we should seek solutions to the paradoxes of modal predicates in the same spirit as in the case of truth.

Unless we are prepared to restrict ourselves to converse wellfounded accessibility relations, we need to adapt and tweak possible worlds semantics. Stern (2016) discussed various strategies. First, one can apply the usual techniques known from the semantic paradoxes. In particular, classical logic can be abandoned. Halbach and Welch (2009) employed Kripke's (1975) fixed-point semantics for their possible worlds semantics without really defending it as a real solution.

Alternatively, condition (ii) of Definition 9.3.2 can be weakened: We do no longer stipulate for all sentences that $\operatorname{Nec}\ulcorner\varphi\urcorner$ holds at $w$ iff $\varphi$ holds in all accessible worlds; we merely stipulate this for certain sentences. Condition (ii) should be satisfied at least for all those sentences $\varphi$ that are expressible with an operator $\square$. This guarantees that the possible worlds semantics for predicates is not 'worse' than possible worlds semantics for the operator $\square$. The class of sentences expressible with an operator $\square$ can be defined as follows using a recursively defined mapping $I$ from the language with the operator $\square$ to the language with the corresponding predicate Nec. Since we deal with de re modality, we need to take care of the free variables in the scope of $\square$. Pick some fixed variable assignment $a_{0}$. The operation that changes the assignment of a given variable $v_{n}$, so 
that the object $x$ is assigned to $v_{n}$ can be expressed in the object language and I write $a_{0}\left(x /\left\ulcorner v_{n}\right\urcorner\right)$ for this operation.

(i) If $\varphi$ does not contain $\square$, then $I(\varphi)=\varphi$.

(ii) $I(\varphi \wedge \psi)=I(\varphi) \wedge I(\psi), I(\neg \varphi)=\neg I(\varphi), I(\forall x \varphi)=\forall x I(\varphi)$, and so on.

(iii) $I\left(\square \varphi\left(x_{1}, \ldots, x_{n}\right)\right)=\operatorname{Nec}\left(\ulcorner I(\varphi)\urcorner, a_{0}\left(x_{1} /\left\ulcorner x_{1}\right\urcorner, \ldots, x_{n} /\left\ulcorner x_{n}\right\urcorner\right)\right)$, where the assignment $a_{0}\left(x_{1} /\left\ulcorner x_{1}\right\urcorner, \ldots, x_{n} /\left\ulcorner x_{n}\right\urcorner\right)$ is obtained by iterated application of the operation mentioned above. Only finitely many such applications are required, because $\varphi$ contains only finitely many variables.

This embedding of the operator languages into the language with a predicate is well-known, and its origin can be traced back at least to Carnap (1934, IV.B.e). ${ }^{6}$ Here I have only added the free variables. The class of formulae expressible with an operator $\square$ is now simply the set of all $I(\varphi)$ such that $\varphi$ is a formula of the language with the operator $\square$ only. If condition (ii) of Definition 9.3.2 is restricted to such sentences, there are pw-models for arbitrary frames.

Sentences and formulae generated with Gödel's diagonal construction cannot be obtained with $I$ from sentences of operator modal logic, but also not general formulx of the form $\forall x(\chi(x) \rightarrow \mathrm{Nec} x)$. Some but not all formulae of the latter kind can be unproblematic and be included as permissible instances of condition (ii) of Definition 9.3.2.

A more comprehensive class of permissible instances of condition (ii) of Definition 9.3.2 can be obtained by singling out the 'grounded' formulae and sentences, defined along the lines of Kripke's (1975). If we apply this account to formulæ in general, the notion of groundedness relative to a variable assignment will have to be defined. In the brief following comments I restrict myself to sentences. The groundedness approach has the disadvantage that the set of such sentences is no longer definable in the object language (under fairly general assumptions). Moreover, whether a sentence is grounded may depend on contingent factors. If all pw-models with this restricted condition (ii) are considered, many general principles such as the distribution of necessity over $\rightarrow$ in pw-models without dead ends have to be abandoned; they will only hold for grounded sentences. This flies in the face of the predicate approach: After all, I adopted the predicate approach in order to express quantified principles. Not being able to get them as quantified generalizations seems odd. At any rate, the desirable models may be sought among those that are pw-models in the weaker sense of satisfying (ii) only for grounded sentences. A different option may be to introduce a new primitive predicate for determinacy or groundedness into the language as in Fujimoto and Halbach (2019) for truth.

An elegant and philosophically useful way to obtain generalization without compromising too much on the expected properties of possible 
worlds semantics is given by Stern (2014a,b, 2016). The idea is to state at least quantified principles with a truth predicate, as we routinely do in informal philosophical discourse. For this strategy, however, a sophisticated theory of truth will be required that avoids the paradoxes.

The challenge of the paradoxes arises for de dicto and de re modalities, although some paradoxes require quantification. In particular, McGee's $\omega$-inconsistency (1985) and the Yablo-Visser paradox are of this kind (Visser, 1989; Yablo, 1985, 1993). A binary predicate may lend itself to the study of these paradoxes. Finally, the move to a binary predicate can be used to recover diagonalization and other syntactic operations without any explicit syntax-theoretic axioms (Halbach and Zhang, 2016; Halbach and Leigh, 2021). I will not pursue this topic here, but rather turn to the application of the fourth grade of modal involvement to an issue in metaphysics.

\subsection{A Problem for Actualism}

In this section I illustrate my claim that using the fourth grade of modal involvement can shed new light on topics and discussions in metaphysics, using an argument by Bealer (1993). In many ways the predicate conception of modalities is a more versatile framework for modal metaphysics than first-order quantified modal logic. The ontology of the objects to which the modalities are ascribed is not hidden away in the metatheory. The expressive power generated by the predicate conception permits an analysis of many questions in the object theory instead of the metatheory, which is usually set-theoretic and extensional.

For instance, it has become common to assume that propositions, as the objects that can be necessary, do not exist in a possible world; as sets of possible worlds, they live in the 'modal æther' (Forster, 2005) and are thereby incomparable to other normal objects. On the predicate conception, we talk about the objects that can be necessary in the object language, and we have to decide whether they exist in a world, as sets of worlds, or wherever. In the outline above they exist in all worlds. But this is only one option; moreover, they are assumed to have the structure of formulae. A metaphysician more serious about proposition may structure them in a different way. Of course, the ontology of propositions has received much attention, and I will not pursue this topic here. Instead I focus on the second argument place of the binary modal predicate Nec, the variable assignments. When they are discussed in the usual settheoretic metatheory, they receive little attention. If they can be talked about in the object language, they become interesting and puzzling. In particular, they can shed new light on the discussion between actualism and possibilism (or possibilism and necessitism in Williamson's 2013 terminology). The semantics outlined above is actualist in the sense that the domains $\mathcal{D}_{w}$ can vary between worlds $w$; at each $w$ the quantifiers 
range only over $\mathcal{D}_{w}$. Possibilist semantics is a special case: Nothing rules out that $\mathcal{D}_{v}=\mathcal{D}_{w}$ for all $v, w \in W$, that is, constant domain semantics is a special case of the actualist semantics.

The argument in this section is not intended as a definitive argument in favour of possibilism or some other position; the section merely demonstrates the effects of injecting objects, in particular, variable assignments from the metalanguage into the object language and how this move can shed light on metaphysical questions.

Bealer (1982, 1993) and others have used arguments of the kind discussed in this section to argue for an ante rem conception of universals. I could follow Bealer here, but in the present setting-which is different from Bealer's-it would mean that variable assignments exist prior to their elements. There would be variable assignments that assign nonexisting objects to some variables, which is hardly acceptable.

What is going to follow is a partial recantation of Halbach and Sturm (2004). The discussions there and Bealer's (1993) paper suffer from the absence of formal semantics. With a framework such as the one outlined above this family of puzzles can now be discussed in a more rigorous way.

As I mentioned already, in quantified modal logic variable assignments are used only in the set-theoretic, extensional metatheory, where questions about possibilism and actualism cannot arise. In our semantics the variable assignments are pushed into the object language that contains modalities. In the presence of modality, the theory of sequences (and sets) becomes more complicated in actualist semantics: Sequences exist only at a world, if all its members exist at that world. This assumption is built into our semantics. In the following example our semantics may yield an unexpected result because of this assumption.

There is a planet, and instead of this planet another planet could have existed which could have coexisted with the first.

There may be more than one reading of this sentence. The reading I have in mind, can be made explicit using possible worlds: In our world $w_{1}$ there is a planet $A$ and there is a possible world $w_{2}$ accessible from our world where $A$ does not exist, but planet $B$ does; moreover, there is another world $w_{3}$ accessible from $w_{2}$ where both planets $A$ and $B$ exist. The reading is captured by the following formalization in quantified modal logic:

$$
\exists x(\operatorname{Pla} x \wedge \diamond(\neg \exists z z=x \wedge \exists y(\text { Pla } y \wedge \diamond(\exists z z=x \wedge \exists z z=y))))
$$

The formula $\exists z z=x$ expresses that $x$ exists. That is, $\exists z z=x$ becomes false at a world $w$ under a variable assignment $a$ if $a(x) \notin \mathcal{D}_{w}$. This is 
the case for our semantics for $\mathrm{Nec}$, and we assume that the same holds for the possible worlds semantics for the operator $\square$.

A minimal possible worlds model $\mathcal{M}$ of (PLAD) looks as follows:

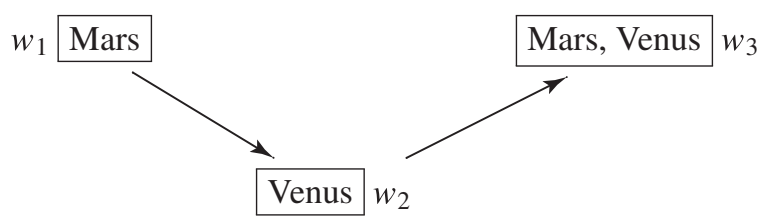

The three worlds of the model are related by the accessibility relation as in the diagram. The domain of $w_{1}$ is \{Mars\}, and so on for the other worlds. The unary predicate Pla $x$ applies at a world to all objects that exist in that world. It is easily seen that then (PLAD) is true at $w_{1}$ in this model.

The crucial feature of the example is that the first quantifier $\exists x$ binds an occurrence of $x$ in the scope of two possibility operators $\diamond$. The witness of the existentially quantified sentence (PLAD) at $w_{1}$ is Mars, which exists at $w_{1}$, but not in the next world $w_{2}$; it exists again in the third world $w_{3}$. In operator modal logic this does not pose a problem; it is not required that $x$ exists in the intermediate world $w_{2}$ for the existential quantifier $\exists x$ to bind an occurrence of $x$ in the scope of two modal operators. As I will show, however, it does cause a problem if a modal predicate is used. The formalization of (PLA) with a binary predicate Nec instead of the modal operator $\square$ is false at $w_{1}$ in the pw-model corresponding to the model $\mathcal{M}$ above, while (PLAD) is true at $w_{1}$, as pointed out above.

To substantiate my claim that the predicate formalization gives a different truth value, I specify the predicate formalization of PLA and the (predicate) pw-model corresponding to the operator model $\mathcal{M}$ above.

First, I sketch how to transform $\mathcal{M}$ into a pw-model $\langle W, R, I, B\rangle$ for the language with Nec instead of $\square$. $W$ and $R$ stay the same. The domain $\mathcal{D}_{w}$ for one of the three worlds $w \in W$ is obtained by adding all syntactic objects to the domain of $w$ in $\mathcal{M}$ and then closing under the formation of sequences. Thus $\mathcal{D}_{w_{1}}$ contains Mars, but not Venus, $\mathcal{D}_{w_{2}}$ only Venus, while $\mathcal{D}_{w_{3}}$ contains both. Consequently, $\mathcal{D}_{w_{2}}$ does not contain any sequences involving Mars. Pla applies at $w$ exactly to all planets in $w$. The existence of such a model is guaranteed by lemma 9.3.3 above, because the accessibility relation is converse wellfounded.

The formalization of (PLA) with the model predicate Nec looks as follows, if $\operatorname{Pos}(x, y)$ is an abbreviation for $\neg \operatorname{Nec}(\neg x, y)$ where $\neg$ represents the function of negating a sentence. Thus, Pos stands for possibility.

$\exists x\left(\operatorname{Pla} x \wedge \operatorname{Pos}(\underbrace{\left\ulcorner\neg \exists z z=x \wedge \exists y\left(\operatorname{Pla} y \wedge \operatorname{Pos}\left(\left\ulcorner\exists z z=x \wedge \exists z z=y^{\urcorner},\left\langle\frac{x}{\left\ulcorner x^{\urcorner}\right.}, \frac{y}{\left\ulcorner y^{\urcorner}\right\urcorner}\right\rangle\right)\right)\right.\right.},\left\langle\frac{x}{\left\ulcorner x^{\urcorner}\right\rangle}\right\rangle)\right)(\mathrm{N})$ 
The expression $\left\langle\frac{x}{r_{x\urcorner}}, \frac{y}{r^{7}}\right\rangle$ in the underbraced quotation name and the less complex $\left\langle\frac{x}{r x\urcorner}\right\rangle$ require an explanation. I assume that the vocabulary permits to describe the variable assignment assigning an object to a specific variable. Here I have treated $\left\langle\frac{x}{x_{1}}, \frac{y}{y_{1}}\right\rangle$ like a function expression with four free variables $x, x_{1}, y$, and $y_{1}$ expressing the function that gives applied to objects $x$ and $y$ and variables $x_{1}$ and $y_{1}$ the relevant variable assignment; but this may have to circumscribed if no suitable function symbol is available. In addition, we need the quotation function sending an expression (here a variable) to a name for that expression. In arithmetical contexts the numeral function serves this purpose.

Since there are no further free variables, a variable assignment of length 2 in the underbraced name suffices. As mentioned above, $\neg$ is stipulated to be the value of all other variables. The 'outer' variable assignment $\left\langle\frac{x}{r x\urcorner}\right\rangle$ has only length 1 , because the preceding formula in corners has only $x$ free. In $\frac{x}{\left.\Gamma_{x}\right\urcorner}$ the upper occurrence of $x$ is used, while $\ulcorner x\urcorner$ is only a mentioning of the same variable; of course, different variables could be used. If the bound variable $x$ in the formula above is renamed as $v$ and the relativization to planets is omitted for readability, the following formula is obtained:

$$
\exists v \operatorname{Pos}\left(\left\ulcorner\neg \exists z z=x \wedge \exists y \operatorname{Pos}\left(\ulcorner\exists z z=x \wedge \exists z z=y\urcorner,\left\langle\frac{x}{\ulcorner x\urcorner}, \frac{y}{\ulcorner y\urcorner}\right\rangle\right)\right\urcorner,\left\langle\frac{v}{\ulcorner x\urcorner}\right\rangle\right)
$$

Of course, any occurrences in the quotation name are not affected by the renaming and $x$ is retained.

In a nutshell, the reason why $(\mathrm{N})$ fails at $w_{1}$ is the following: If $(\mathrm{N})$ were true at $w_{1}$, there would have to be a variable assignment in $\mathcal{D}_{w_{2}}$ that assigns Mars to $x$ and Venus to $y$. But there is no such variable assignment in $\mathcal{D}_{w_{2}}$, because Mars does not exist in $w_{2}$, that is Mars is not an element of $\mathcal{D}_{w_{2}}$. In fact, such a variable assignment exists only at $w_{3}$ where both, Mars and Venus exist.

A more explicit version of this argument can be given as follows. Let $\langle W, R, I, B\rangle$ be the pw-model described above. To show that $(\mathrm{N})$ fails at $w_{1}$, assume to the contrary that it is true at $w_{1}$. Since Mars is the only potential witness of the existential quantifier, also the following must hold:

$$
\begin{aligned}
\langle W, R, I, B\rangle \models_{w_{1}} & \operatorname{Pos}(\ulcorner\neg \exists z z=x \wedge \exists y(\text { Pla } y \wedge \\
\operatorname{Pos}(\ulcorner\exists z z=x & \left.\left.\left.\left.\wedge \exists z z=y\urcorner,\left\langle\frac{x}{\ulcorner x\urcorner}, \frac{y}{\ulcorner y\urcorner}\right\rangle\right)\right)\right\urcorner,\left\langle\frac{x}{\ulcorner x\urcorner}\right\rangle\right) \quad\left[\left\langle\frac{\text { Mars }}{\ulcorner x\urcorner}\right\rangle\right]
\end{aligned}
$$

That is, we assume that the formula Pos(...) holds at world $w_{1}$ under the variable assignment that assigns Mars to $x$. The expression in square brackets is the variable assignment in the metalanguage, for which we conveniently use the same notation as in the object language. It follows from the assumption 
that the formula denoted by the quotation name must be satisfied by Mars for $x$ at the world accessible from $w_{1}$ :

$$
\begin{aligned}
&\langle W, R, I, B\rangle \models_{w_{2}} \neg \exists z z=x \wedge \exists y(\text { Pla } y \wedge \\
&\left.\operatorname{Pos}\left(\ulcorner\exists z z=x \wedge \exists z z=y\urcorner,\left\langle\frac{x}{\ulcorner x\urcorner}, \frac{y}{\ulcorner y\urcorner}\right\rangle\right)\right) \quad\left[\left\langle\frac{\text { Mars }}{\ulcorner x\urcorner}\right\rangle\right]
\end{aligned}
$$

Hence both conjuncts must hold at $w_{2}$. The first conjunct expresses that $x$ does not exist. Because Mars fails to be in $\mathcal{D}_{w_{2}}$, the first conjunct does hold indeed.

The second conjunct is an existentially quantified sentence. The only witness that cold make the second conjunct true is Venus, because the witness must be a planet and Venus is the only planet that exists in $\mathcal{D}_{w_{2}}$. Therefore the following must obtain:

$$
\langle W, R, I, B\rangle \models_{w_{2}} \operatorname{Pla} y \wedge \operatorname{Pos}\left(\ulcorner\exists z z=x \wedge \exists z z=y\urcorner,\left\langle\frac{x}{\ulcorner x\urcorner}, \frac{y}{\ulcorner y\urcorner}\right\rangle\right) \quad\left[\left\langle\frac{\text { Mars }}{\ulcorner x\urcorner}, \frac{\text { Venus }}{\ulcorner y\urcorner}\right\rangle\right]
$$

This implies the following:

$$
\langle W, R, I, B\rangle \models_{w_{2}} \exists z z=\left\langle\frac{x}{\ulcorner x\urcorner}, \frac{y}{\ulcorner y\urcorner}\right\rangle \quad\left[\left\langle\frac{\text { Mars }}{\ulcorner x\urcorner}, \frac{\text { Venus }}{\ulcorner y\urcorner}\right\rangle\right]
$$

That is, at $w_{2}$ there is a finite sequence containing Mars and Venus, contrary to our assumption that finite sequences in a world can contain only objects existing in that world. Therefore, the initial assumption is refuted and, therefore, ( $\mathrm{N}$ ) fails at $w_{1}$, while the operator version (PLA口) holds at $w_{1}$ in the corresponding operator model $\mathcal{M}$. Intuitively, the operator version yields the expected result, while the predicate version does not.

The problem for actualism is not caused by any restrictions on the accessibility relation. It cannot be avoided by replacing the accessibility relation $R$ above with its transitive closure. We cannot have a total accessibility relation because of Theorem 9.3.4; but even if diagonalization were banned and total accessibility relations permitted, the problem for actualism would remain.

Various strategies to address the problem were discussed by Bealer (1993) and Halbach and Sturm (2004). I do not provide a thorough discussion, but only sketch some of them.

First, an actuality operator or predicate cannot overcome the problem: The variable assignment assigning Mars to $x$ and Venus to $y$ could be claimed to exist in the actual world $w_{1}$ instead of the world $w_{2}$; but the variable assignment cannot exist at $w_{1}$ either, because Venus is not in $w_{1}$. Bealer (1993) made this observation already for universals rather than variable assignments.

The second strategy sounds desperate: Variable assignment assigning non-existing objects to variables could be permitted; variable assignments 
would exist ante rem. Such entities would be true creatures of darkness. I certainly could not think of them as functions from the set of variables into the domain of the world or as any mathematical entities. However, stranger entities have been dreamt in philosophy. If the point argument is made about universals instead of variable assignment, as Bealer (1993) does, an argument for ante rem universals is obtained.

Thirdly, actualism could be rejected in favour of possibilism, that is, constant domain semantics. The same objects exist in all worlds, but only some are 'instantiated'. Variable assignments could be formed using uninstantiated objects.

Finally, one could use a proxy $b$ in $w$ for an object $a$ that does not exist at $w$ and form variable assignments with proxies. All the worlds of a pwmodel have an infinite domain, so this is not obviously ruled by cardinality constraints. ${ }^{7}$

I do not take a stance here. The problem just outlined is supposed to demonstrate how the additional expressive strength permits a discussion of issues of metaphysics in the object language. In first-order quantified modal logic the problem does not arise, because it is moved entirely into the metalanguage and there problems are solved by using a purely extensional, non-modal metatheory. One may even consider replacing modal talk with purely extensional discourse about possible worlds, as Lewis $(1968,1986)$ did. Of course, I cannot analyze such alternative approaches here, but one of the criteria would be whether they reach the expressive strength of the fourth grade of modal involvement.

\subsection{The Road Ahead}

I have outlined one way to capture the strength of the fourth grade of modal involvement in a formal language containing a binary predicate $\mathrm{Nec}$ and with a possible worlds semantics. But this is only one way to capture the full strength of the fourth grade of modal involvement. There are various ways to deviate from the approach in this chapter, and I have only briefly sketched some reasons for my choices. However, I hope I have succeeded in conveying a taste of how the fourth grade of modal involvement can help to shed light on issues such as the discussion between actualists and possibilists. The fourth grade of modal involvement may not open a Cantorian paradise, but at least it provides an expansive playground not only for metaphysics, but also for the analyses of various epistemic, alethic, logical, and further modalities.

\section{Acknowledgments}

I am grateful to Beau Mount, Simon Nagler, Carlo Nicolai, Johannes Stern, Philip Welch, and Timothy Williamson for useful hints and suggestions. 


\section{Notes}

1. Quine used 'Nec' (uppercase) for the predicate and 'nec' (lowercase) for the operator. Here the latter is replaced with the more familiar $\square$.

2. For a recent detailed discussion of modal predicates see Stern (2016). It has been argued that the full force of modal discourse can be restored by using propositional or 'substitutional' quantification or truth predicates (Halbach and Welch, 2009). Some remarks on such extensions are given in the next section.

3. Here I refer to "Three Grades of Modal Involvement" only. Later, in Word and Object $(1960, \S 41)$ he considers de re modalities as predicates; and earlier in $\$ 35$ and in (1956) he discusses propositional attitudes de re.

4. Instead of predicates of sentences we could use predicates of Russellian propositions. If we are able to express the operation of applying the property $P$ to the object $o$, we can use a predicate to say that there is a property $P$ and an object $O$ such that the proposition resulting of applying $P$ to $O$ is necessary. This approach has its own problems, because now de dicto modalities are not directly expressible. I will not pursue this strategy any further without claiming that it is not viable. A more detailed discussion would require a formalized theory of Russellian propositions.

5. When Quine talks about a ordering of grades of modal involvement, he does not have an ordering according to reducibility in our sense in mind. As mentioned above, a semantic predicate is the lowest form of modal involvement for Quine.

6. Carnap's translations is different and there are several variations. What caused problems especially in early variants were problems with the iteration of Nec.

7. I thank Beau Mount for bringing this point to my attention.

\section{References}

Asher, N. and Kamp, H. (1989). Self-reference, attitudes, and paradox. In Chierchia, G., Partee, B. H., and Turner, R., editors, Properties, Types and Meaning, volume 1, pages 85-158. Kluwer.

Bealer, G. (1982). Quality and Concept. Clarendon Press.

Bealer, G. (1993). Universals. Journal of Philosophy, 90: 5-32.

Bealer, G. (1998). Universals and properties. In Laurence, S. and Macdonald, C., editors, Contemporary Readings in the Foundations of Metaphysics, pages 131-147. Blackwell Publishers.

Bull, R. (1969). On modal logic with propositional quantifiers. Journal of Symbolic Logic, 34: 257-263.

Carnap, R. (1934). Logische Syntax der Sprache. Springer.

Fine, K. (1970). Propositional quantifiers in modal logic. Theoria, 36: 336-346.

Forster, T. (2005). The modal aether. In Kahle, R., editor, Intensionality, pages 20-41, A K Peters.

Fujimoto, K. and Halbach, V. (2019). Classical Determinate Truth. Draft.

Garson, J. (2001). Quantification in modal logic. In Handbook of Philosophical Logic, pages 267-323. Kluwer Academic Publishers.

Halbach, V. and Leigh, G. (2021). The Road to Paradox: A Guide to Syntax, Truth, and Modality. Cambridge University Press, reprinted in Martin.

Halbach, V., Leitgeb, H., and Welch, P. (2003). Possible worlds semantics for modal notions conceived as predicates. Journal of Philosophical Logic, 32: 179-223. 
Halbach, V. and Sturm, H. (2004). Bealers Masterargument: Ein Lehrstück zum Verhältnis von Metaphysik und Semantik. Facta Philosophica, 6: 97-110.

Halbach, V. and Welch, P. (2009). Necessities and necessary truths: A prolegomenon to the metaphysics of modality. Mind, 118: 71-100.

Halbach, V. and Zhang, S. (2016). Yablo without Gödel. Analysis, 76: 53-59.

Kaplan, D. (1970). S5 with quantifiable propositional variables. Journal of Symbolic Logic, 35: 355. Abstract.

Kripke, S. A. (1959). A completeness theorem in modal logic. Journal of Symbolic Logic, 24: 1-14.

Kripke, S. A. (1975). Outline of a theory of truth. Journal of Philosophy, 72: 690-716. reprinted in Martin (1984).

Lewis, D. (1968). Counterpart theory and quantified modal logic. Journal of Philosophy, 65: 113-126.

Lewis, D. (1986). On the Plurality of Worlds. Blackwell Publishers. Malden, MA.

Martin, R. L., editor (1984). Recent Essays on Truth and the Liar Paradox. Clarendon Press and Oxford University Press.

McGee, V. (1985). How truthlike can a predicate be? A negative result. Journal of Philosophical Logic, 14: 399-410.

Montague, R. (1963). Syntactical treatments of modality, with corollaries on reflexion principles and finite axiomatizability. Acta Philosophica Fennica, 16: 153-167. Reprinted in (Montague, 1974, 286-302).

Montague, R. (1974). Formal Philosophy: Selected Papers of Richard Montague. Yale University Press. Edited and with an introduction by Richmond $\mathrm{H}$. Thomason.

Quine, W. V. O. (1956). Quantifiers and propositional attitudes. Journal of Philosophy, 53: 177-187.

Quine, W. V. O. (1960). Word and Object. MIT Press. Cambridge, MA.

Quine, W. V. O. (1976). Three grades of modal involvement. In The Ways of Paradox, pages 158-176. Harvard University Press, Cambridge, MA, revised and enlarged edition.

Stern, J. (2014a). Modality and axiomatic theories of truth I: Friedman-Sheard. Review of Symbolic Logic, 7: 273-298.

Stern, J. (2014b). Modality and axiomatic theories of truth II: Kripke-Feferman. Review of Symbolic Logic, 7: 299-318.

Stern, J. (2016). Toward Predicate Approaches to Modality, volume 44 of Trend in Logic. Springer.

Tarski, A. (1935). Der Wahrheitsbegriff in den formalisierten Sprachen. Studia Philosophica Commentarii Societatis Philosophicae Polonorum, 1: 261-405. Translated as 'The Concept of Truth in Formalized Languages' in (Tarski, 1956, 152-278); page references are given for the translation.

Tarski, A. (1956). Logic, Semantics, Metamathematics: Papers from 1923 to 1938. Clarendon Press.

Visser, A. (1989). Semantics and the liar paradox. In Gabbay, D. and Günthner, F., editors, Handbook of Philosophical Logic, volume 4, pages 617-706. Reidel, Dordrecht.

Williamson, T. (2013). Modal Logic as Metaphysics. Oxford University Press.

Yablo, S. (1985). Truth and reflection. Journal of Philosophical Logic, 14: 297-349.

Yablo, S. (1993). Paradox without self-reference. Analysis, 53: 251-252. 